\title{
面向数据排名的可视分析方法综述
}

\author{
周志光 ${ }^{1,2)}$, 程奥圣 ${ }^{1)}$, 朱申吉 ${ }^{1)}$, 李国俊 1$)$, 梅晓蔚 $)^{*}$ \\ 1) (浙江财经大学信息管理与人工智能学院 杭州 310018) \\ 2) (浙江大学 CAD\&CG 国家重点实验室 杭州 310058) \\ (meixiaowei151@163.com)
}

\begin{abstract}
摘 要: 根据属性关键字的大小对数据对象进行排名, 能够帮助用户快速而精准地判断和决策. 文中对面向数据排 名的可视分析进行综述. 首先从映射表示大小关系的视觉元素出发, 介绍坐标轴位置、长度、角度、面积和亮度/饱 和度等视觉元素在排名可视化过程中的设计与应用; 然后从数据结构形式出发, 介绍多维、时序、空间和拓扑等面向 数据排名的可视分析方法; 再概述面向数据排名的可视分析技术在经济金融、城市交通和文体传媒等领域的应用研 究; 最后总结面向数据排名的可视分析面临的挑战, 并对未来工作进行了展望.
\end{abstract}

关键词：排名规则；排名可视化; 可视分析; 交互式排名

中图法分类号: TP391.41ＤOI: 10.3724/SP.J.1089.2021.19264

\section{A Survey on the Visual Analytics for Data Ranking}

\author{
Zhou Zhiguang ${ }^{1,2)}$, Cheng Aosheng ${ }^{1)}$, Zhu Shenji ${ }^{1)}$, Li Guojun ${ }^{1)}$, and Mei Xiaowei ${ }^{1{ }^{*}}$ \\ 1) (School of Information Management and Artificial Intelligence, Zhejiang University of Finance and Economics, Hangzhou 310018) \\ 2) (State Key Laboratory of CAD\&CG, Zhejiang University, Hangzhou 310058)
}

\begin{abstract}
Ranking is a popular and universal approach to sort items based on the value of its attributes, which can make judicious and informed decisions effectively. This paper reviews the related research on the visual analysis for data ranking. Firstly, the design and application of visual elements such as coordinate axis location, length, angle, area and brightness/saturation from the perspective of visual element mapping is introduced. Secondly, with different structural forms of data for ranking, an overview of the advanced technologies and methods with respect to multidimensional, temporal, spatial and topological features is proposed. Furthermore, applications of ranking visual analysis in the human economy, urban traffic, culture, sports and entertainment are investigated. Finally, the challenges and future developments of ranking visualization are prospected.
\end{abstract}

Key words: ranking techniques; ranking visualization; visual analytic; interactive ranking

排名常用于描述数据对象的大小关系, 可以 帮助人们快速而准确地判断和决策, 被广泛应用 于经济金融 ${ }^{[1]}$ 、城市交通 ${ }^{[2]}$ 文体和传媒 ${ }^{[3]}$ 等领域, 如市场商品价格排名、旅游景点人气指数排名、体

\section{育赛事球队积分排名等.}

排名的本质是根据属性关键字的大小关系对 数据对象进行排序. 经典的排名方法通常是依赖 于单一属性对数据对象进行排名, 如 Demuth ${ }^{[4]}$ 提

收稿日期：2021-07-07; 修回日期：2021-08-15. 基金项目：国家自然科学基金(61872314); 教育部人文社会科学研究项目 (18YJC910017); 浙江省高校重大人文社科攻关计划(2018QN021); 浙江省科技厅公益项目(LGF20F020010, LGF20G010003); 浙江省 自然科学基金(LY18F020024); 浙江大学 CAD\&CG 国家重点实验室开放课题(A2001). 周志光(1983一), 男, 博士, 教授, CCF 会员, 主要研究方向为数据可视化与可视分析; 程奥圣(1997一), 男, 硕士研究生, 主要研究方向为数据可视化与可视分析; 朱申吉 (2000一), 女, 在校学生, 主要研究方向为数据可视化与可视分析; 李国俊(1998一), 男, 硕士研究生, 主要研究方向为数据可视化与 可视分析; 梅晓蔚(1992-), 女, 博士, 讲师, CCF 会员, 论文通讯作者, 主要研究方向为数据可视化与可视分析. 
出的冒泡排序、Shell ${ }^{[5]}$ 提出的插人排序以及 Hoare ${ }^{[6]}$ 提出的快速排序等. 基于单一属性的排名 算法原理和过程比较简单, 其核心任务更多聚焦 于排名效率的提升. 然而, 当数据对象的结构或属 性关系复杂, 如数据对象具有时空属性或网络结 构关系时, 排名目标和过程不再简单, 且排名结果 难以和数据对象建立直观的联系, 从而导致排名 的过程和结果难以分析和理解, 甚至在具体场景 中复杂问题的分析和解决过程中存在不确定性或 矛盾.

大量学者面向关系复杂的数据开展了创新性的 排名方法研究. 例如, 机器学习方法用于多维属性 数据排名(learning to rank, LTR), 包括: (1) PointWise ${ }^{[7]}$, 即将多维属性数据排名转换为回归问题; (2) ListWise $^{[8]}$, 利用完整的数据输人列表训练模 型; (3) PairWise ${ }^{[9-10]}$, 从用户排名的数据子集中导 出成对约束来训练模型. 又如 PageRank 模型 ${ }^{[11]}$, 其对具有网络结构关系的数据对象进行排名. 在 此基础上，相关学者提出了更多的基于 PageRank 的优化排名方法 ${ }^{[12-13]}$, 用于提升网络结构中节点 重要性的排名精度.

然而, 由于属性及结构关系复杂, 数据对象的 排名过程不够直观, 排名结果难以与数据对象的 属性和结构建立关联, 给进一步理解和应用排名 方法带来限制. 近年来, 可视化领域学者提出多种 有效的排名可视分析方法, 利用直观的图形展现 复杂数据对象的排名过程及其与结果之间的联系, 有效地增强了排名结果的认知, 并进一步拓展了 排名方法的应用. 为帮助可视化领域学者和业界 用户更加方便、高效地调研和理解排名可视分析方 法, 进而在不同的数据分析场景中合理地选择和 使用, 本文从排名可视化中的视觉元素、复杂结构 数据排名可视分析、排名可视分析应用 3 个方面, 对面向数据排名的可视分析方法进行了综述. 首 先, 对排名可视化中的视觉元素进行总结, 对于常 用于大小关系表达的视觉元素进行梳理, 包括坐 标轴位置、长度、角度、面积、亮度/饱和度等; 进 而, 从复杂的数据结构特征角度出发, 分别介绍面 向具有多维、时序、空间、网络等复杂结构特征的 排名可视化和交互分析方法; 最后, 对排名可视分 析方法在不同场景中的应用进行了调研和概述.

\section{1 排名可视化中的视觉元素}

排名可视化是数据对象属性大小关系的直观
呈现. 陈为等学者在《可视化导论》 ${ }^{[14]}$ 一书中提出, 坐标轴位置、长度、角度、面积、颜色亮度/饱和 度等是常见的用于表达事物、对象大小关系的视觉 元素, 而这些视觉元素也广泛用于面向数据排名 的可视化设计.

坐标轴位置表现数据对象大小关系更加直观, 易于理解. VisMatchmaker ${ }^{[15]}$ 利用散点图中点的位 置差异, 直观地表示对象的受欢迎程度及其收人 情况. 可视分析系统 $\mathrm{TelCoVis}^{[16]}$ 利用平行坐标轴 上的位置关系, 直观地表达和比较不如图 1 所示同 类别特征在属性轴上的分布.

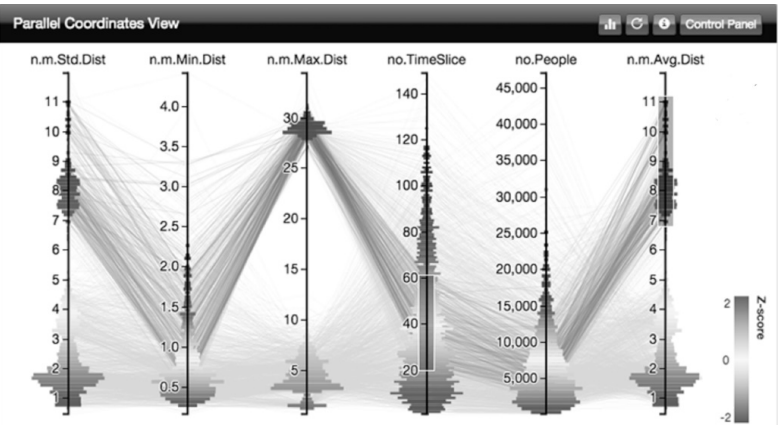

图 1 平行坐标系中位置分布表达数据对象大小关系 ${ }^{[16]}$

连线长度和角度是呈现排名关系的有效方法. Weng 等 ${ }^{[17]}$ 将条形图嵌人排名视图中的每列, 一列 代表一个属性, 条形图的高度编码了一定范围内 与该属性匹配的候选对象的数量. $\mathrm{Xu}$ 等 ${ }^{[18]}$ 利用线 条的角度表示装配线的生产情况, 斜率变化较大 的线条更加明显，方便用户快速地感知和捕捉异 常模式, 如图 2 所示.

图形大小是映射排名关系的常用方法. Viégas 等 ${ }^{[19]}$ 将关键词按照一定顺序和规律排名, 如频度 递减、字母顺序等, 进而以文字的大小代表词语的 重要性. $\mathrm{Xu}$ 等 ${ }^{[20]}$ 利用词云描述主题竞争情况, 单 词的大小与相应主题的竞争力正相关, 如图 3 所 示. $\mathrm{Pu}$ 等 ${ }^{[21]}$ 展示用户组在基站中的移动模式, 圆的 半径大小编码底层基站中用户组的通话次数.

颜色亮度/饱和度也是表示数据对象大小关系 的有效方法. Shi 等 ${ }^{[22]}$ 用亮度映射数据大小, 在地 图中显示美国大学课程 GT 的学习者的空间分布情 况. Correll 等 ${ }^{[23]}$ 利用亮度/饱和度映射加拿大各个区 域犯罪事件密度的大小, 如图 4 所示, 南部省份的 犯罪密度明显较大，表明这些省份的安全性较低。

用户自定义专题图案设计方法广泛用于排名 可视化. Wang 等 ${ }^{[24]}$ 提出 InfoNice, 设计装载不同量 级的酒瓶示意性地表达葡萄酒的消费量大小，如 


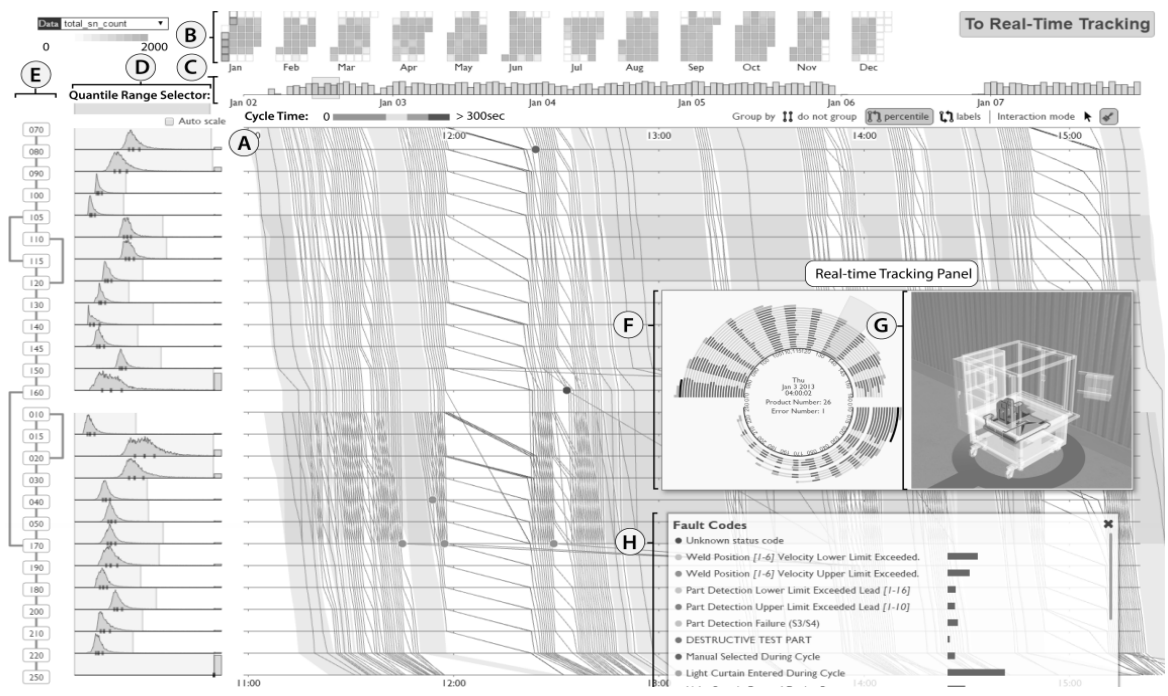

图 2 线条斜率大小表现机器状态差异 ${ }^{[18]}$

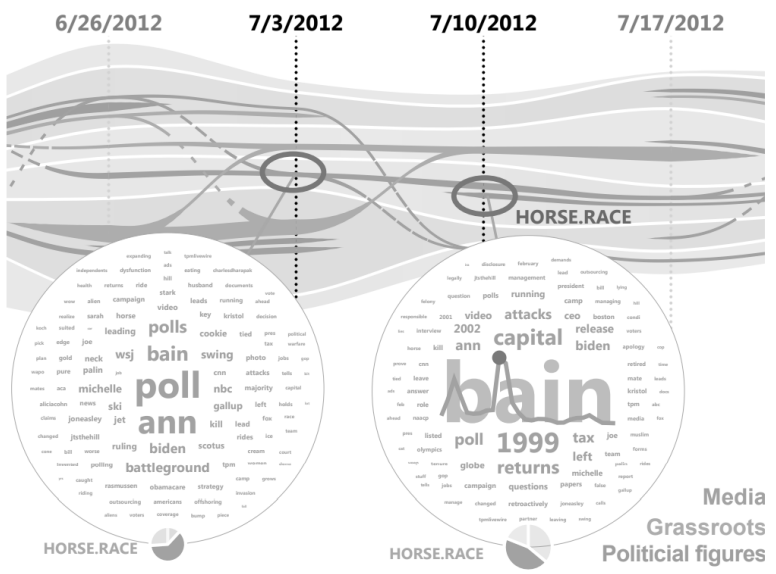

图 3 单词的大小编码排名 ${ }^{[20]}$

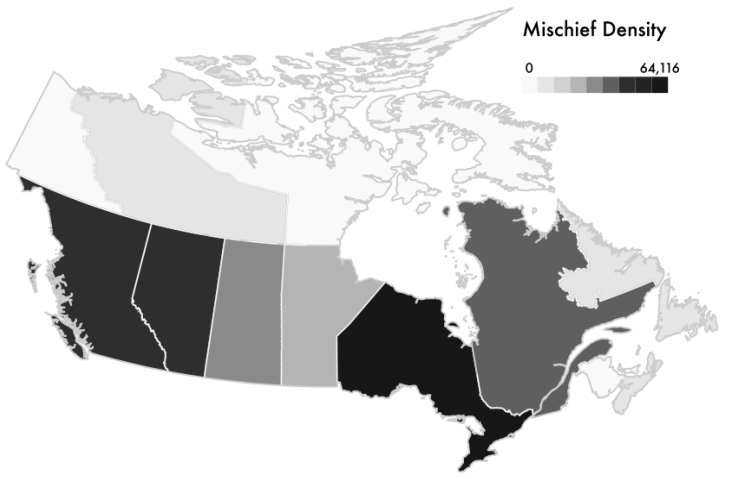

图 4 加拿大各区域犯罪事件密度 ${ }^{[23]}$

图 5 所示. Perin 等 ${ }^{[25]}$ 在排名表中添加图标编码、彩 色圆圈等嵌人式可视化图形，以应对排名列表中 由于项目过多而造成的视觉混乱问题. Andrienko 等 ${ }^{[26]}$ 设计新的排名视觉元素符号，将每个对象的 排名嵌人有条形图或饼图的地图中，符号中填充 部分越大，排名越高.

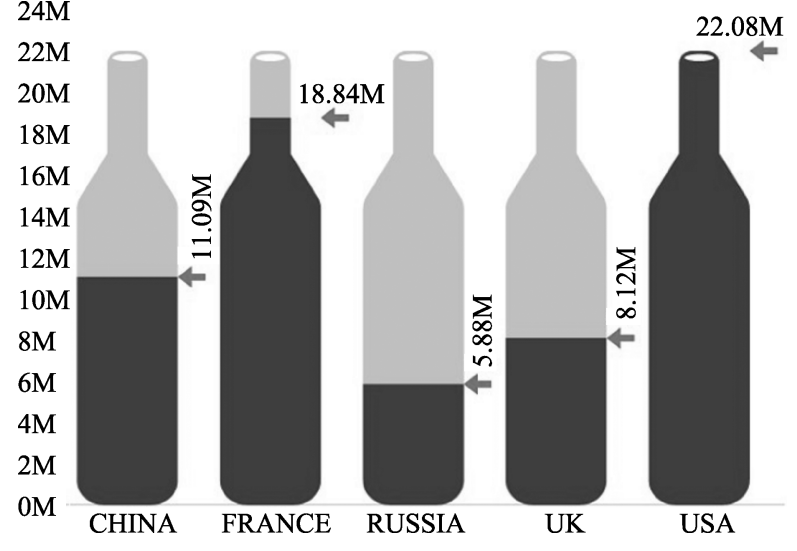

图 5 InfoNice 自定义图案编码排名 ${ }^{[24]}$

\section{2 复杂结构数据排名可视分析}

实际场景中，数据对象普遍具有多维、时序、 空间、网络拓扑等复杂结构特点, 传统排名算法和 可视化设计在数据理解和结果分析过程中存在局 限性. 可视化领域工作者从不同的数据结构角度 出发，提出有针对性的排名可视分析方法.

\section{1 多属性数据排名可视分析}

数据对象通常具有多维属性, 如大学影响力 排名的各种指标属性、食物多种营养成分含量和宜 居城市的多种因素排名对比等. 针对单一属性进 行排名简单且容易理解, 而考虑多维属性, 给出合 理且有说服力的综合排名结果是一项困难的任务. 可视分析是展现不同属性对于综合排名结果的贡 献, 以帮助用户理解和交互探索多属性数据排名 结果的有效途径.

为解决排名项目过多导致可视化结果杂乱的 
问题, RankExplorer ${ }^{[27]}$ 对排名进行分组并使用主题 河流图可视化数据时变趋势, 同时结合颜色条和 字形对排名的变化进行编码. Behrisch 等 ${ }^{[28]}$ 使用 Small Multiples 方法进行多个排名的可视分析利用
径向节点链接字形可视化不同排名之间的相似性 和差异性. Gratzl 等 ${ }^{[29]}$ 设计 LineUp, 利用堆叠的条 形图比较不同的对象, 并结合斜率图直观地解释多 维属性权重的改变如何影响最终排名, 如图 6 所示.

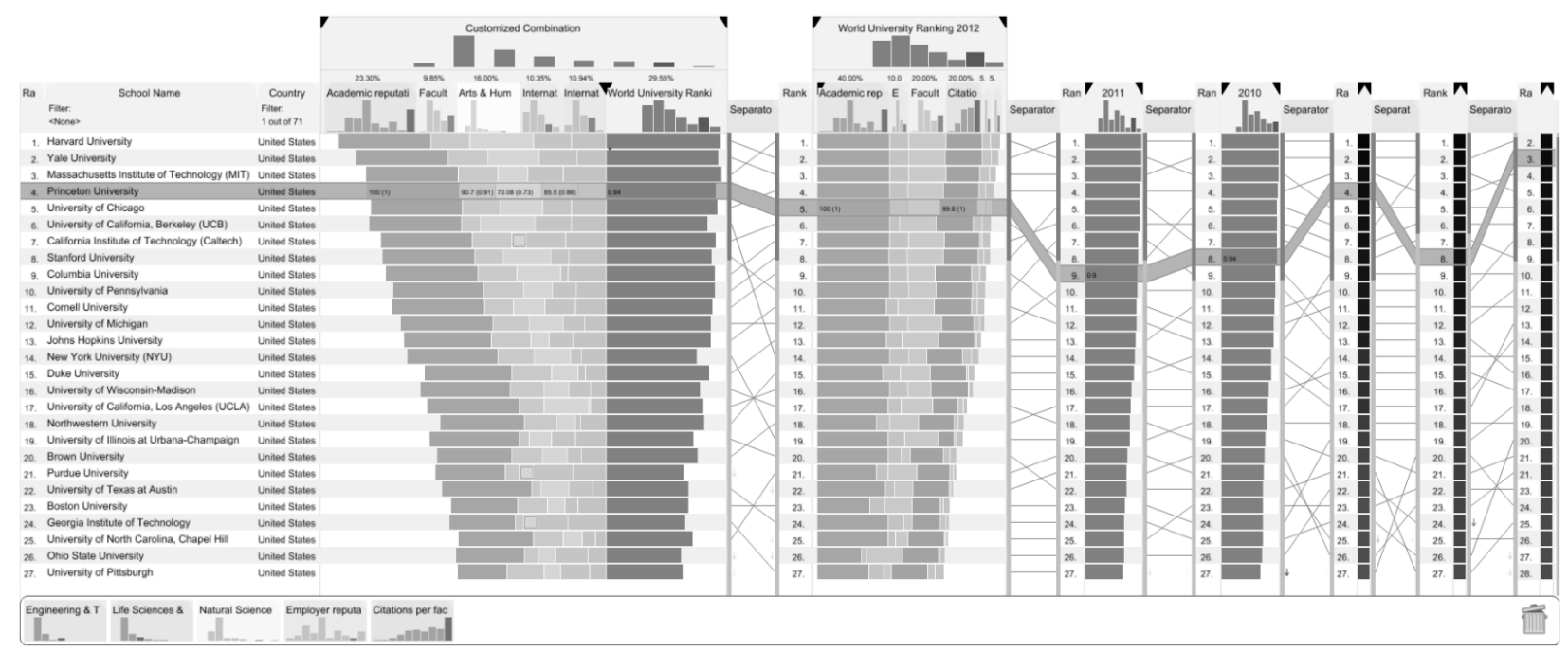

图 6 LineUp 系统界面 ${ }^{[29]}$

Pajer 等 ${ }^{[30]}$ 设计 WeightLifter 利用权重加权求 和进一步探索分析属性权重空间，帮助用户能够 更好地理解决策对权重变化的敏感性，进而提高 多准则决策效率和综合决策的可信度. 为探索大 量不完整排名和部分排名数据, Kidwell 等 ${ }^{[31]}$ 通过 多维尺度热图进行可视化, 针对不同属性组合的 排名结果存在明显的差异性, 设计多种可视化技 术对多属性数据进行排名对比分析.

为结合用户对数据排名关系的主观认知，进 一步理解潜在的复杂多维数据排名及其变化原因, 交互技术已成为多维属性排名可视分析的重要手 段. 图 7 所示的 Podium ${ }^{[32]}$ 系统支持用户交互地改变 表行, 根据用户对数据对象的认知进行排名, 进而 采用排名支持向量机推断出一个权重模型，尽可能 地满足用户的数据偏好. ValueCharts ${ }^{[33]}$ 和 LineUp ${ }^{[29]}$ 允许用户通过单击和拖动以交互地调整用于排名的 属性权重自定义地创建排名, 用户可以快速地查看

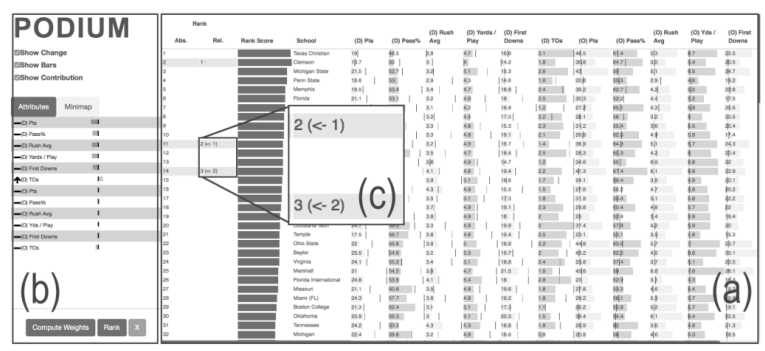

图 7 Podium 系统界面 ${ }^{[32]}$
更改属性权重如何影响数据的排名结果. 在 rankby-feature 框架 ${ }^{[34]}$ 中, 用户可以选择一个排名标准, 对多维数据集在单个属性维度或所有可能的两两 组合属性维度, 依据所选的排名标准来对属性进 行排名, 从而深人探索分析多维数据属性的潜在 关系.

\section{2 时间序列排名可视分析}

与一般的排名可视化不同，时间序列排名可 视化表示随着时间演化的数据排名情况, 其数据 对象包含时间属性, 不仅要表达数据随时间变化 趋势, 还要展示出数据排名变化的规律. 时间序列 数据排名分析在金融和商业等许多应用中发挥着 重要作用.

Vuillemot 等 ${ }^{[35]}$ 提出了一种时间导航技术, 通 过用户直接操作更新排名表. 这种技术允许用户 拖动表格的单元格更改时间段, 而折线图则覆盖 在表格的顶部展示更改的情况，也是控制数据更 新速度的视觉提示. 为了呈现螺旋状的排名趋势, Batty ${ }^{[36]}$ 采用了径向坐标表示不同时间点的排名. RankExplorer ${ }^{[27]}$ 将排名时间序列自适应地划分为 多个组, 使用颜色和字形扩展主题河流图, 并提供 交互方式用于检查不同对象排名之间的相互关系 以及每个对象自身排名的变化情况，如图 8 所示. Xia 等 ${ }^{[37]}$ 使用了几种视觉设计显示排名数据的时 间演变, 如图 9 所示, 通过将排名带或河流图分解 为分散的字形避免视觉混乱. RankEvo ${ }^{[38]}$ 通过计算 
排名项目时间序列之间的相似性, 将相似的项目 组织成项目集, 进而探索排名的演变以及排名列 表的异常目. LiveRAC ${ }^{[39]}$ 在基于网格的布局中显示 了时间序列数据的多个视图, 因此用户可以轻松 地在多个细节级别上排名比较不同的序列. 如图 10 所示, Han 等 ${ }^{[40]}$ 提出了一种基于热图和直方图的 视觉编码方案支持用户通过悬停、刷选和过滤等交 互技术，在大规模的时间排名集合中探索数据时 间序列的分布和不确定性.

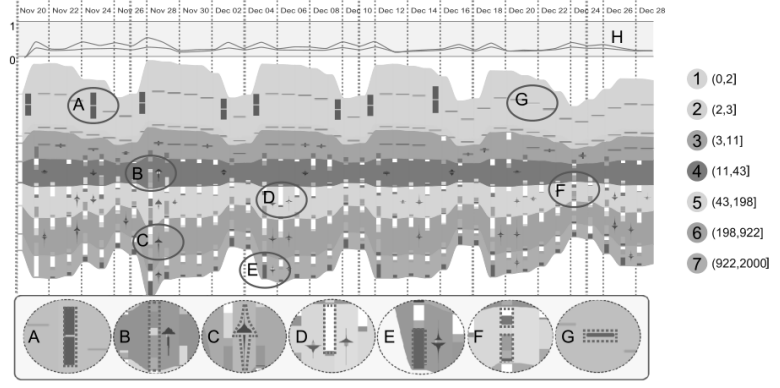

图 8 RankExplorer 系统界面 ${ }^{[27]}$

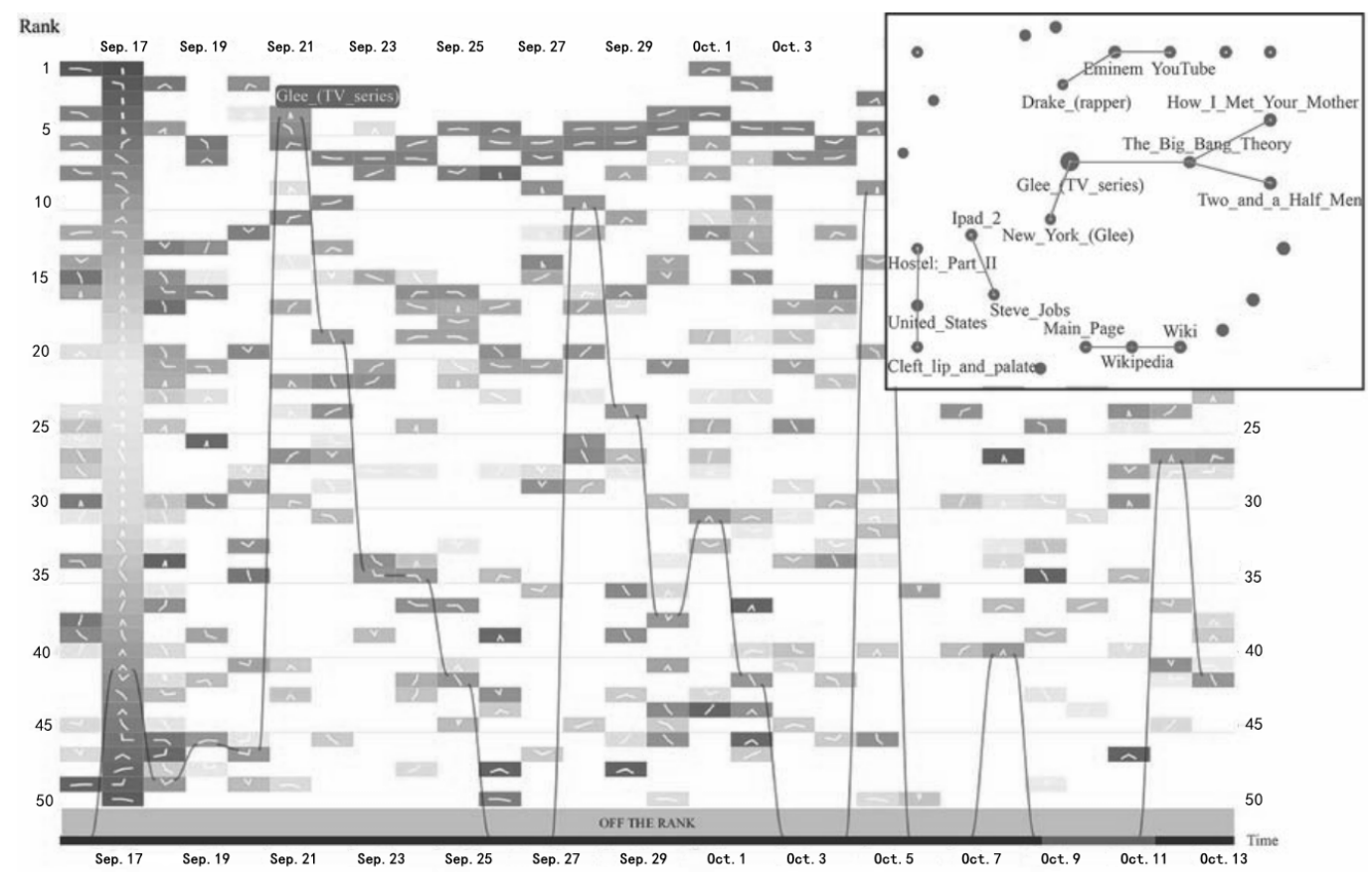

图 9 维基百科每周电视连续剧排行榜的演变趋势 ${ }^{[37]}$
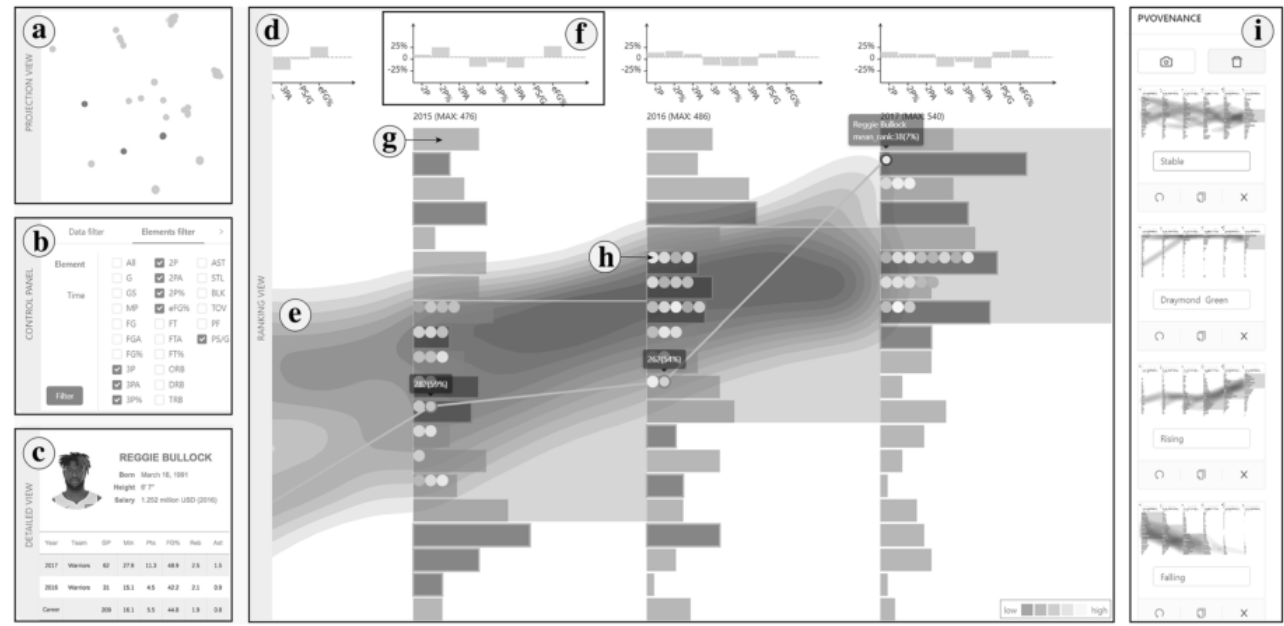

图 10 RankBrushers 系统界面 ${ }^{[40]}$

\section{3 空间结构排名可视分析}

空间对象存在固定的位置关系，因此难以借助 经典的可视化设计表达排名关系. Andrienko 等 ${ }^{[26]}$ 提
出了实用符号, 可以将每个对象的排名嵌人带有条 形图或饼图的地图中，以有效地分析空间排名可视 结果. Nusrat 等 ${ }^{[41]}$ 根据属性的大小对地图区域边界 
的形状进行形变, 更清楚地展示排名结果. 此外, 多窗口视图和协同交互技术常用于大规模空间数据 的排名分析. Weng 等 ${ }^{[17]}$ 提出了基于点的 SRVis 技术, 使用可扩展的基于矩阵的可视化技术和堆积条形图 对大规模空间数据排名并对排名原因进行编码, 成 功地将排名与空间上下文集成, 并且不存在严重的 视觉混乱, 如图 11 所示. Liu 等 ${ }^{[42]}$ 设计交互式可视 化分析系统 SmartAdP, 结合可视化驱动的数据挖掘 模型, 通过多组视图对广告牌位置的候选方案进行 排名, 并从不同层级深人地探索分析候选方案之间 的内在关联关系，识别共性和差异以帮助广告策划
者更好地决策. Cao 等 ${ }^{[43]}$ 基于张量的非监督异常分 析算法, 结合可视化和交互设计开发了系统框架 Voila, 依据用户先验知识对异常判断的实时反馈, 引导算法更加高效、精准地识别时空数据异常模式, 实现实时在线异常监测, 同时对多个疑似异常的区 域进行排名和记录以供后续进一步排查. Tra$j \operatorname{Rank}^{[44]}$ 分析车辆同一路线的多条运行轨迹, 根据 交通运行花费时间对轨迹进行排名分组，同时还展 示了轨迹运行时间随出行时间点的变化, 从时间排 名视图、空间视图和盒图等多个视角探索车辆沿同 一路线的行驶行为, 如图 12 所示.

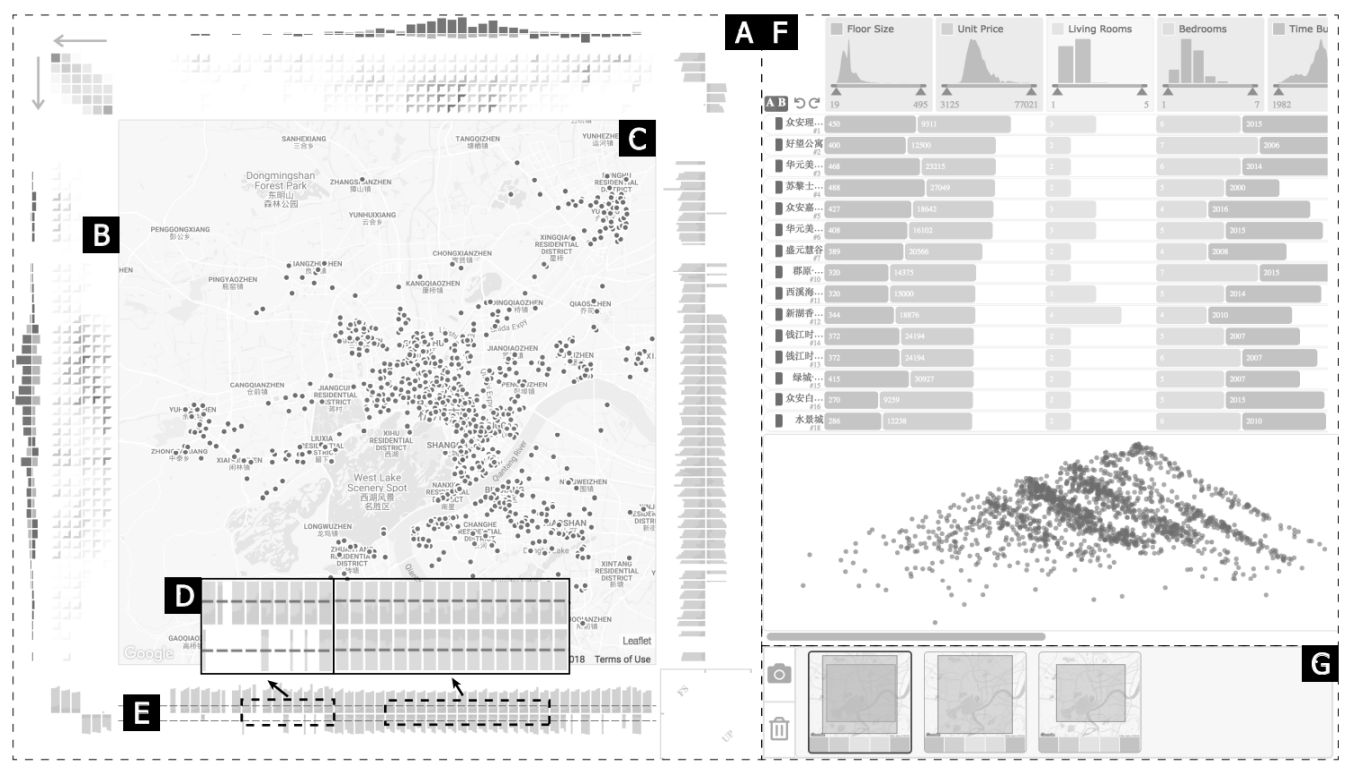

图 11 SRVis 系统界面 ${ }^{[17]}$
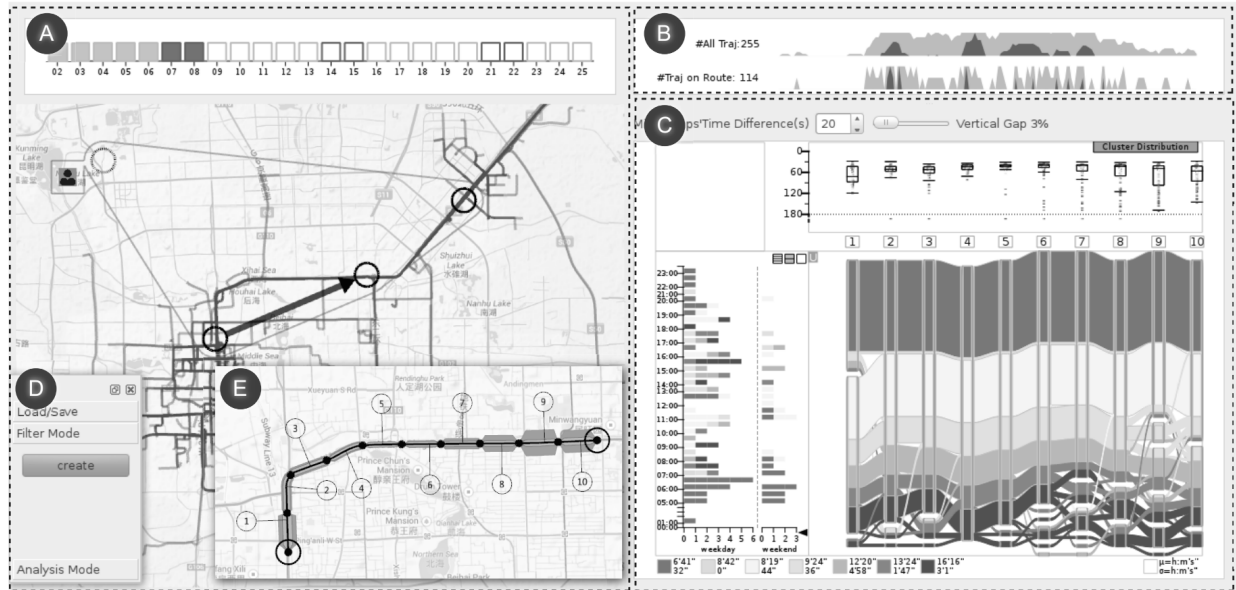

图 12 TrajRank 系统界面 ${ }^{[44]}$

\section{4 拓扑结构排名可视分析}

拓扑结构是数据对象之间常见的关系特征, 通常借助点和线描述数据对象之间的拓扑关系. 拓扑网络结构中，节点的排名是一个经典的工作
方向, 如 PageRank 等.

可视化领域往往通过更改点线链接图中节点和 连边的外观, 如大小、颜色、纹理和形状等, 同步展 示拓扑结构信息和属性信息 ${ }^{[45]}$. 对于节点, 颜色和 
大小是最常用的映射元素, Cao 等 ${ }^{[46]}$ 开发的 g-Miner 可以在多元图数据上对组进行可视化挖掘, 节点代 表公司员工, 并设计成饼状图, 扇区的颜色和大小 分别表示技能的类型和员工的能力水平, 如图 13 所 示. 在连边映射中, 研究人员通常通过修改线条的 宽度、颜色和曲率等表达关系属性变化. 例如, $\mathrm{Wu}$ 等 ${ }^{[47]}$ 提出以个人为中心的作者合作网络可视化方法
egoSlider, 如图 14 所示, 通过 glyph 设计和连边粗细 映射展示感兴趣用户的合作信息随时间的变化情况.

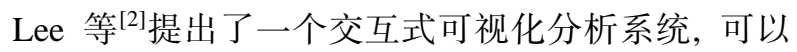
基于车辆检测器数据进行交通拥堵探索、监视和预 测, 将道路流量和流速通过线条的宽度和颜色进行 映射, 并设计了表格视图按速度、流量和每条道路 的估计行驶时间对道路进行排名等，如图 15 所示.
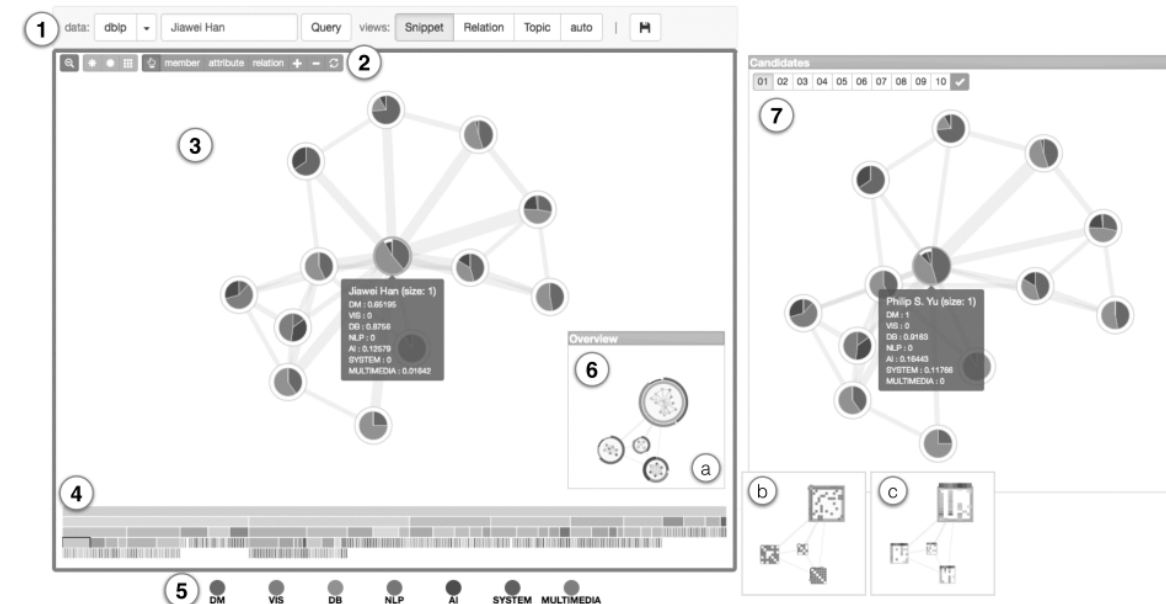

图 13 g-Miner 系统界面 ${ }^{[46]}$

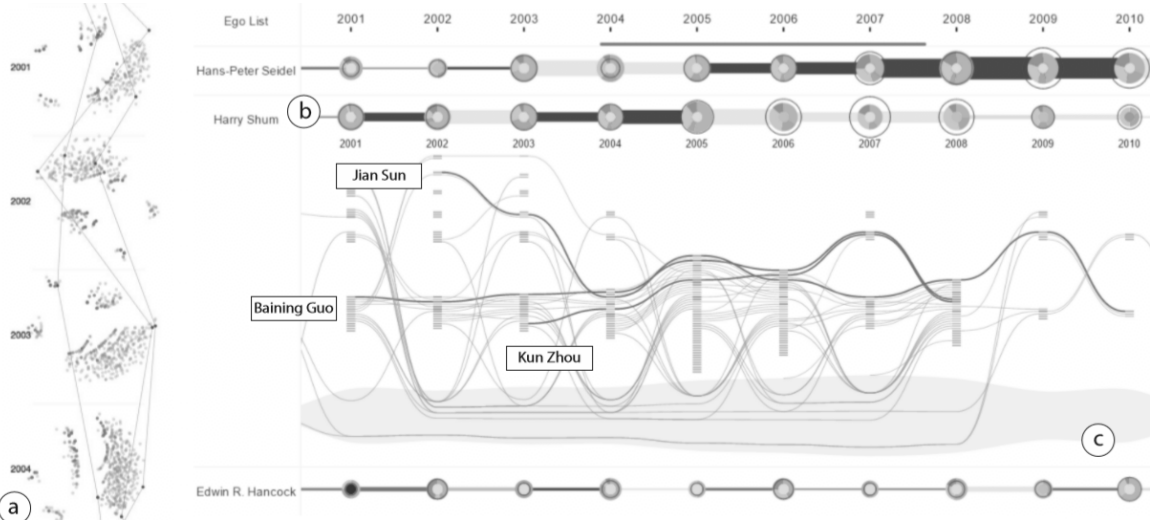

图 14 作者合作网络可视分析系统界面 ${ }^{[47]}$

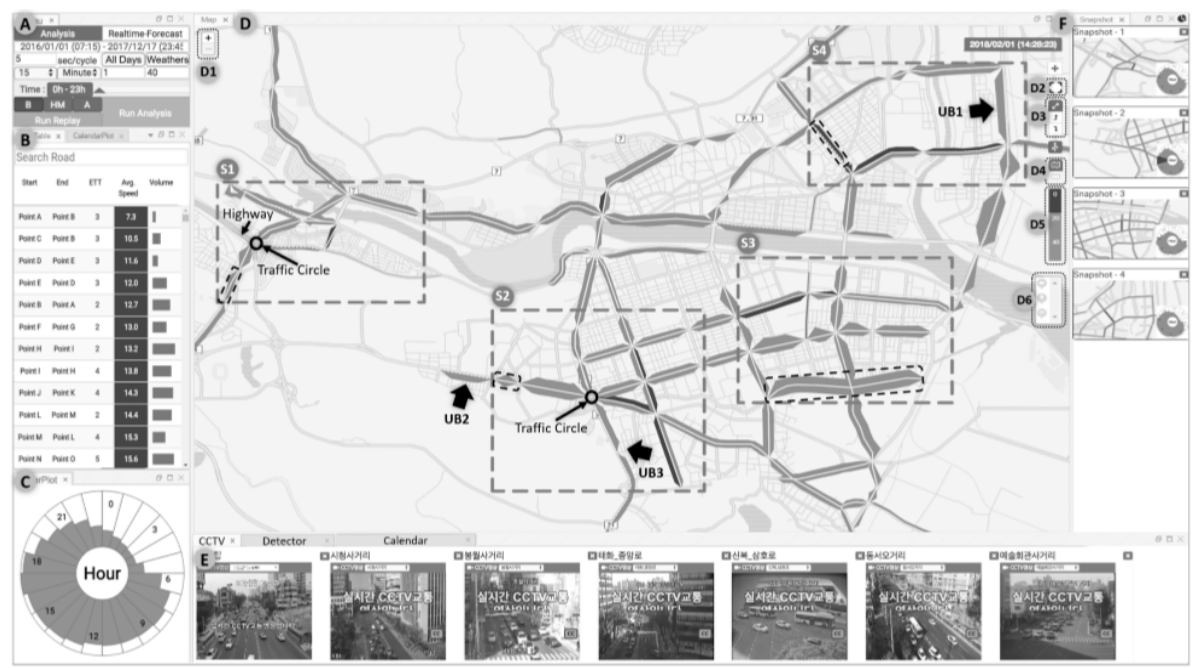

图 15 交通拥堵可视分析系统界面 ${ }^{[2]}$ 
为进一步深人探索网络拓扑结构特征的排名, Shen 等 ${ }^{[88]}$ 在一个异构网络中呈现社交和空间信息 分析社交团体的聚集地，其中餐厅、体育馆和公园 等位置节点分别使用不同的颜色表示，节点越大， 与之相连的社交用户在该位置聚集的频率越高. Zhou 等 ${ }^{[12]}$ 采用 Word2Vec 模型将文章转换为高维 向量来表征引文的上下文语义关系，然后用余弦 相似度公式计算转换为向量的引文网络中文章之 间的相似性, 以此作为影响权重对 PageRank 模型 进行优化，从而对文章学术影响力进行排名. Wang 等 ${ }^{[49]}$ 设计引文网络可视化工具 ImpactVis, 如图 16 所示, 左右两侧的柱状图表示当前学者的论文和 论文被引用的数量, 通过线条连接与之相关的学 者, 以此帮助专家分析发表论文和引用论文的数 量与质量对学者学术排名的影响.

\section{3 排名可视分析应用}

排名可视分析能够有效地帮助用户快速的选
择和精准的决策，被广泛应用于各个领域，如经济 金融、城市交通、文体传媒等.

\section{1 经济金融}

排名可视分析在经济金融领域得到了广泛的 应用. 例如, 工作人员利用柱状图长度映射价格排 名情况，利用折线图映射股票一段时间内增长情 况，以及现在词云的提出对金融行业可视分析提 供了巨大的帮助. 随着电子商务的快速发展, 越来 越多的在线评论网站, 如 Yelp, 帮助客户做出更好 的购买决策. 为了解决在线评论数量巨大, 用户评 级标准存在潜在差异, 以及评论的时间、长度、细 节和质量等各有不同的问题, Yong 等 ${ }^{[50]}$ 介绍了基 于在线评论的精心设计的可视化分析系统 E-Comp, 如图 17 所示, 用以帮助客户不同详细程 度地对本地企业实现快速和全面的比较. Shi 等 ${ }^{[27]}$ 开发的 RankExplorer 可视化分析 1995-2010 年美 国财富 500 强数据, 并找到企业排名下降的原因. Weng 等 ${ }^{[17]}$ 提出了空间排名可视化技术 SRVis, 将 排名与空间情境整合探索可供出售的房屋, 并根

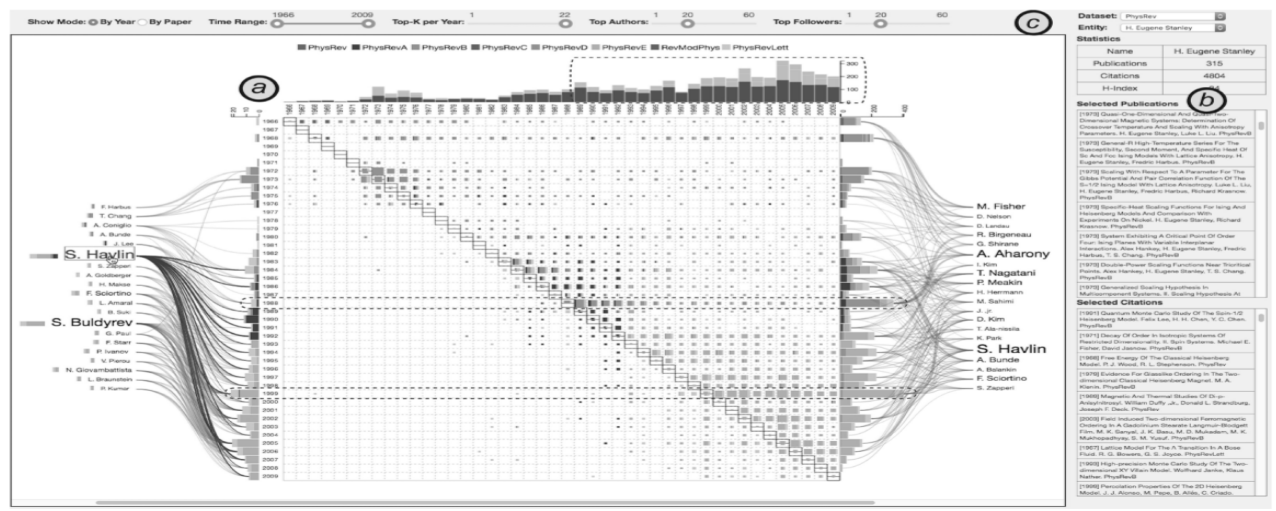

图 16 引文网络可视分析系统 ${ }^{[49]}$

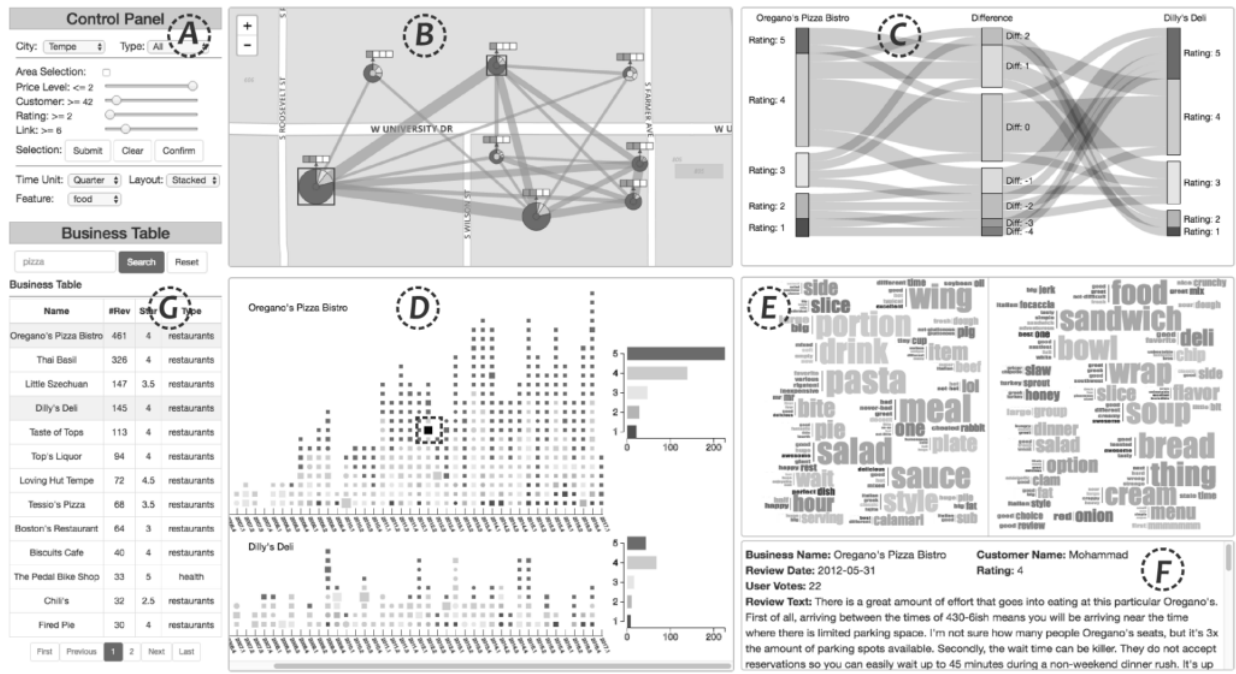

图 17 E-Comp 系统的用户界面 ${ }^{[50]}$ 
据客户的要求向其提供更好的房屋建议. Agryzkov 等 ${ }^{[1]}$ 将城市网络中每个地点不同类型商业活动的 真实信息视作节点, 利用 PageRank 算法根据节点 在网络中的重要性对所有节点进行排名, 以此可 视化一个城市的某些商业活动并帮助城市研究者 和规划者了解经济活动在城市区域内的分布.

\section{2 城市交通}

排名可视分析在城市运营中扮演重要角色. 在应对城市交通问题方面，学者通常采用大小、颜 色、位置等视觉元素表达城市中站点、拥堵程度等 排名情况. Lee 等 $^{[2]}$ 开发的可视分析系统，可以排
名拥堵路段的堵塞程度、拥堵时长, 帮助城市规划 人员更好地进行交通管制和路线规划. 如图 18 所 示, Cao 等 ${ }^{[43]}$ 开发的系统框架 Voila 可以同时对多 个疑似异常的区域进行排名和记录，帮助城市交 通管理者识别分析交通模式的异常情况并及时采 取预防措施. 地理位置规划选择已成为城市规划 的关键, Liu 等 ${ }^{[42]}$ 提出的 SmartAdP 利用出租车轨迹 数据通过多组视图对广告牌位置的候选方案进行 排名, 在城市规划中, 该系统同样适用于其他位置 选择问题，如使用轨迹数据选择商圈或学校的位 置等.

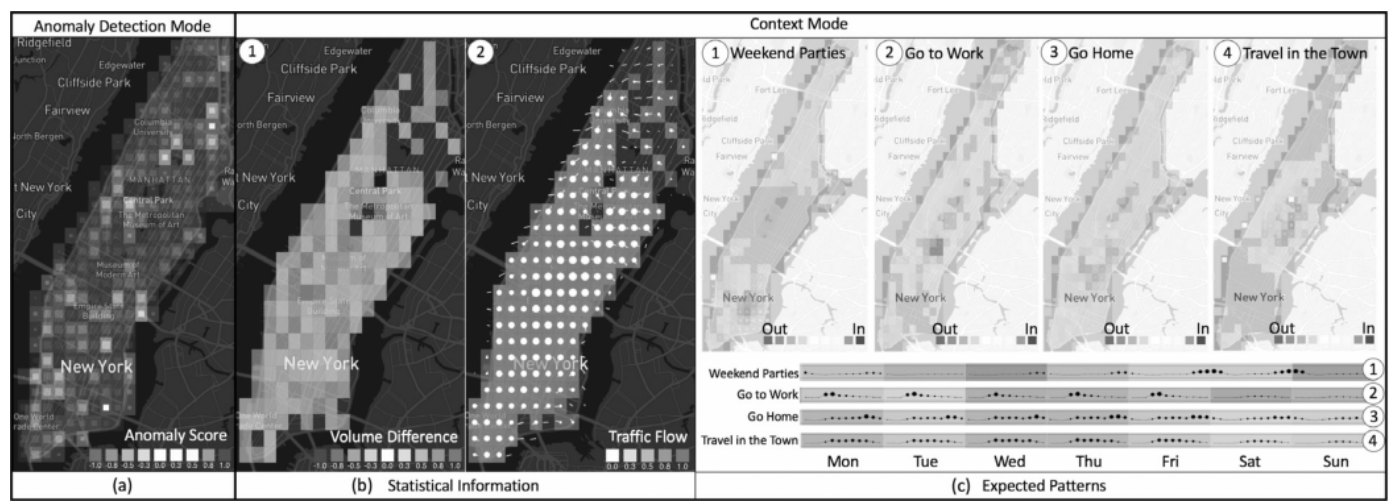

图 18 Voila 系统对异常区域的检测 ${ }^{[43]}$

\section{3 文体传媒}

在文化领域, LineUp ${ }^{[29]}$ 可以很好地对大学进 行排名, 它利用堆叠条形图映射影响大学排名的 各个属性, 并用斜线可视属性改变大学排名的变 化情况. Zhou 等 ${ }^{[12]}$ 提出的系统在对引文网络进行 学术影响力可视化排名方面十分有效. 在体育竞 技方面, Perin 等 ${ }^{[25]}$ 在球队排名表中添加图标编码、 彩色等嵌人式可视化图形，使用户清晰地观察目 标球队的排名变化情况. Chung 等 ${ }^{[3]}$ 提出了基于 glyph 的排名可视化工具, 用于比较和分析橄榄球 比赛中的趋势. 球迷可以通过 Podium ${ }^{[32]}$ 发现最喜 欢球队的重要特征，以帮助球迷预测球队在接下来 的赛季中的排名. Han 等 ${ }^{[40]}$ 设计的 RankBrushers 可 以对 NBA 球员在赛季中的排名进行有效的分析和 跟踪，让用户深人了解随着时间的推移整体排名 的变化, 并寻求变化规律以及解释分析原因. 在传 媒领域, Maheswari 等 ${ }^{[51]}$ 设计的 RankTagViz 根据语 义对用户游记进行排名，并且基于标签和图像可 视化旅游日志，帮助读者获取有价值的旅游信息. 如图 19 所示, Miranda 等 ${ }^{[52]}$ 提出的 TopKube 更容易 对给定地区和时间内最受欢迎的微博标签进行排 名, 捕捉人们关注的时事热点问题. Xia 等 ${ }^{[37]}$ 设计
的 WikiTopReader 允许用户通过将页面排名行为 与页面链接关系联系起来，探索汶览量最高的页 面随时间的变化, 可应用于跟踪新闻或微博热点 话题.

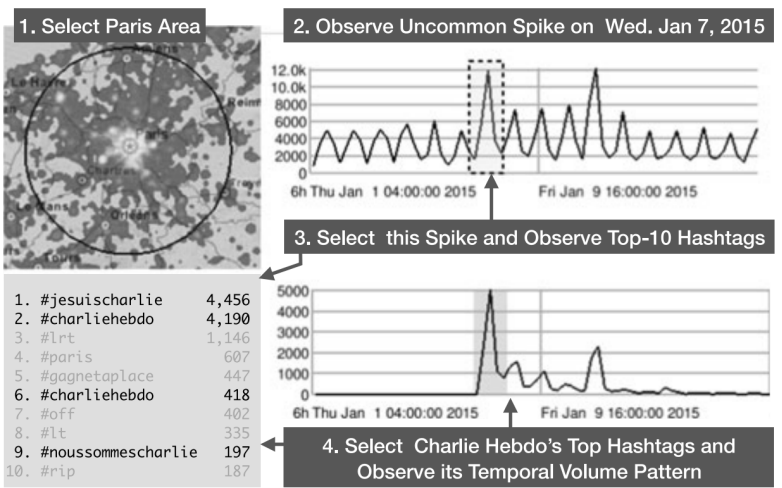

图 19 使用 TopKube 进行微博探索 ${ }^{[52]}$

\section{4 结 语}

面向数据排名的可视分析是可视化领域的研 究热点, 本文对面向数据排名的可视化分析方法 进行了综述. 主要包括映射表示大小关系的视觉 
元素在排名可视化分析中的设计与应用, 面向多 维、时序、空间、网络多种复杂数据结构的排名可 视分析前沿技术和方法，以及排名可视分析技术 在不同行业领域的拓展应用. 随着排名可视化应 用需求的持续增长，排名可视化面向的数据规模 急剧增加, 数据结构也愈加复杂, 结合当前面向数 据排名可视分析的进展，本文总结和探讨了面向 数据排名的可视分析工作仍面临的挑战和未来发 展趋势，具体如下.

(1) 面向数据排名的可视化分析需要具备对 比、分析和解读结构复杂的数据对象排名结果的能 力. 随着数据对象结构的复杂化, 尤其是面临多源 异构、关联关系复杂的数据对象排名和结果解读任 务时，设计有效的可视化方法对排名结果进行探 索和挖掘存在挑战, 是未来的研究趋势.

(2) 面向数据排名的可视化技术需要有效处 理海量大规模数据对象, 实时呈现令人满意的排 名可视化结果. 在面对大规模数据对象时, 现有的 可视化技术对全体数据对象排名结果展示的完整 性和实效性仍难以满足用户需求，如何综合地考 虑对象关系特征，借助各种算法优化加速技术快 速地建立排名关系, 需要进一步深人探索.

(3) 现实场景中, 数据对象之间的排名关系很 难定义简单的比较标准, 因此借助用户的经验和 判断定义排名结果样本, 进而利用机器学习方法 得出满足用户需求的排名结果是可行途径. 因此, 充分发挥人工智能技术的巨大潜能并将其应用于 排名结果可视化, 以寻求有监督或半监督的排名 方法的创新和应用, 是面向复杂结构数据排名方 向的突破点, 需要研究者更深层次的探索.

(4) 面向数据排名的可视化技术缺乏对排名 结果的评估标准. 通常, 评估排名结果的合理性和 有效性, 需要结合领域专家的先验知识和大量的 对比试验或用户评测来度量. 因此, 设计通用的排 名可视化评估标准和体系，帮助用户客观、高效地 认知理解排名可视分析结果，是未来排名可视分 析的重点工作方向.

\section{参考文献(References):}

[1] Agryzkov T, Oliver J L, Tortosa L, et al. Analyzing the commercial activities of a street network by ranking their nodes: a case study in Murcia, Spain[J]. International Journal of Geographical Information Science, 2014, 28(3/4): 479-495

[2] Lee C, Kim Y, Jin S M, et al. A visual analytics system for exploring, monitoring, and forecasting road traffic congestion[J].
IEEE Transactions on Visualization and Computer Graphics, 2020, 26(11): 3133-3146

[3] Chung D H S, Parry M L, Griffiths I W, et al. Knowledge-assisted ranking: a visual analytic application for sport event data[J]. IEEE Computer Graphics and Applications, 2016, 36(3): 72-82

[4] Demuth H B. Electronic data sorting[J]. IEEE Transactions on Computers, 1985, C-34(4): 296-310

[5] Shell D L. A high-speed sorting procedure[J]. Communications of the ACM, 1959, 2(7): 30-32

[6] Hoare C A R. Quicksort[J]. The Computer Journal, 1962, 5(1): 10-16

[7] Cossock D, Tong Z. Subset ranking using regression[C] //Proceedings of the 19th Annual Conference on Learning Theory. Heidelberg: Springer, 2006: 605-619

[8] Liu $\mathrm{T}$ Y. Learning to rank for information retrieval[C] //Proceedings of the 33rd International ACM SIGIR Conference on Research and Development in Information Retrieval. New York: ACM Press, 2010: 197-200

[9] Burges C, Shaked T, Renshaw E, et al. Learning to rank using gradient descent[C] //Proceedings of the 22nd International Conference on Machine Learning. New York: ACM Press, 2005: 89-96

[10] Cao Y B, Xu J, Liu T Y, et al. Adapting ranking SVM to document retrieval[C] //Proceedings of the 29th Annual International ACM SIGIR Conference on Research and Development in Information Retrieval. New York: ACM Press, 2006: 186-193

[11] Page L, Brin S, Motwani R, et al. The PageRank citation ranking: bringing ORDER to the Web[J]. Stanford Digital Libraries Working Paper, 1999, 9(1): 1-14

[12] Zhou Z G, Shi C, Hu M X, et al. Visual ranking of academic influence via paper citation[J]. Journal of Visual Languages \& Computing, 2018, 48: 134-143

[13] Yan E, Ding Y, Sugimoto C R. P-Rank: an indicator measuring prestige in heterogeneous scholarly networks[J]. Journal of the American Society for Information Science and Technology, 2010, 62(3): 467-477

[14] Chen Wei, Zhao Ye, Zhang Song, et al. Introduction to visualization[M]. Beijing: Higher Education Press, 2020: 56-57(in Chinese)

(陈为, 赵烨, 张高, 等. 可视化导论 $[\mathrm{M}]$. 北京：高等教育出 版社, 2020: 56-57)

[15] Law P M, Wu W C, Zheng Y X, et al. VisMatchmaker: cooperation of the user and the computer in centralized matching adjustment[J]. IEEE Transactions on Visualization and Computer Graphics, 2017, 23(1): 231-240

[16] Wu W C, Xu J Y, Zeng H P, et al. TelCoVis: visual exploration of co-occurrence in urban human mobility based on telco data[J]. IEEE Transactions on Visualization and Computer Graphics, 2016, 22(1): 935-944

[17] Weng D, Chen R, Deng Z K, et al. SRVis: towards better spatial integration in ranking visualization[J]. IEEE Transactions on Visualization and Computer Graphics, 2019, 25(1): 459-469

[18] Xu P P, Mei H H, Ren L, et al. ViDX: visual diagnostics of assembly line performance in smart factories[J]. IEEE Transactions on Visualization and Computer Graphics, 2017, 23(1): 291-300

[19] Viégas F B, Wattenberg M, Feinberg J. Participatory visualization with wordle[J]. IEEE Transactions on Visualization and 
Computer Graphics, 2009, 15(6): 1137-1144

[20] Xu P P, Wu Y C, Wei E X, et al. Visual analysis of topic competition on social media[J]. IEEE Transactions on Visualization and Computer Graphics, 2013, 19(12): 2012-2021

[21] Pu J S, Liu S Y, Xu P P, et al. MViewer: mobile phone spatiotemporal data viewer[J]. Frontiers of Computer Science, 2014, 8(2): 298-315

[22] Shi C L, Fu S W, Chen Q, et al. VisMOOC: visualizing video clickstream data from massive open online courses[C] //Proceedings of the IEEE Conference on Visual Analytics Science and Technology. Los Alamitos: IEEE Computer Society Press, 2014: 277-278

[23] Correll M, Heer J. Surprise! Bayesian weighting for de-biasing thematic maps[J]. IEEE Transactions on Visualization and Computer Graphics, 2017, 23(1): 651-660

[24] Wang Y, Zhang H D, Huang R, et al. InfoNice: easy creation of information graphics[C] //Proceedings of the $\mathrm{CHI}$ Conference on Human Factors in Computing Systems. New York: ACM Press, 2018: 1-12

[25] Perin C, Vuillemot R, Fekete J D. A table! improving temporal navigation in soccer ranking tables[C] //Proceedings of the SIGCHI Conference on Human Factors in Computing Systems. New York: ACM Press, 2014: 887-896

[26] Andrienko G, Andrienko N, Jankowski P. Building spatial decision support tools for individuals and groups[J]. Journal of Decision Systems, 2003, 12(2): 193-208

[27] Shi C L, Cui W W, Liu S X, et al. RankExplorer: visualization of ranking changes in large time series data[J]. IEEE Transactions on Visualization and Computer Graphics, 2012, 18(12): 2669-2678

[28] Behrisch M, Bach B, Hund M, et al. Magnostics: image-based search of interesting matrix views for guided network exploration[J]. IEEE Transactions on Visualization \& Computer Graphics, 2017, 23(1): 31-40

[29] Gratzl S, Lex A, Gehlenborg N, et al. LineUp: visual analysis of multi-attribute rankings[J]. IEEE Transactions on Visualization and Computer Graphics, 2013, 19(12): 2277-2286

[30] Pajer S, Streit M, Torsney-Weir T, et al. WeightLifter: visual weight space exploration for multi-criteria decision making[J]. IEEE Transactions on Visualization and Computer Graphics, 2017, 23(1): 611-620

[31] Kidwell P, Lebanon G, Cleveland W S. Visualizing incomplete and partially ranked data[J]. IEEE Transactions on Visualization and Computer Graphics, 2008, 14(6): 1356-1363

[32] Wall E, Das S, Chawla R, et al. Podium: ranking data using mixed-initiative visual analytics[J]. IEEE Transactions on Visualization and Computer Graphics, 2018, 24(1): 288-297

[33] Carenini G, Lloyd J. ValueCharts: analyzing linear models expressing preferences and evaluations[C] //Proceedings of the Working Conference on Advanced Visual Interfaces. New York: ACM Press, 2004: 150-157

[34] Seo J, Shneiderman B. A rank-by-feature framework for interactive exploration of multidimensional data[J]. Information Visualization, 2005, 4(2): 96-113

[35] Vuillemot R, Perin C. Investigating the direct manipulation of ranking tables for time navigation[C] //Proceedings of the 33rd Annual ACM Conference on Human Factors in Computing Systems. New York: ACM Press, 2015: 2703-2706

[36] Batty M. Rank clocks[J]. Nature, 2006, 444(7119): 592-596

[37] Xia J, Hou Y M, Chen Y V, et al. Visualizing rank time series of wikipedia top-viewed pages[J]. IEEE Computer Graphics and Applications, 2017, 37(2): 42-53

[38] Lei H, Xia J, Guo F Z, et al. Visual exploration of latent ranking evolutions in time series[J]. Journal of Visualization, 2016, 19(4): 783-795

[39] McLachlan P, Munzner T, Koutsofios E, et al. LiveRAC: interactive visual exploration of system management time-series data[C] //Proceeding of the 26th International Conference on Human Factors in Computing Systems. New York: ACM Press, 2008: 1483-1492

[40] Han D M, Pan J C, Guo F Z, et al. RankBrushers: interactive analysis of temporal ranking ensembles[J]. Journal of Visualization, 2019, 22(6): 1241-1255

[41] Nusrat S, Alam M J, Kobourov S G. Evaluating cartogram effectiveness[J]. IEEE Transactions on Visualization \& Computer Graphics, 2018, 24(2): 1077-1090

[42] Liu D Y, Weng D, Li Y H, et al. SmartAdP: visual analytics of large-scale taxi trajectories for selecting billboard locations[J]. IEEE Transactions on Visualization \& Computer Graphics, 2017, 23(1): 1-10

[43] Cao N, Lin C G, Zhu Q H, et al. Voila: visual anomaly detection and monitoring with streaming spatiotemporal data[J]. IEEE Transactions on Visualization and Computer Graphics, 2018, 24(1): 23-33

[44] Lu M, Wang Z C, Yuan X R. TrajRank: exploring travel behaviour on a route by trajectory ranking[C] //Proceedings of the IEEE Pacific Visualization Symposium. Los Alamitos: IEEE Computer Society Press, 2015: 311-318

[45] Liu Yuhua, Ni Lushan, Zhou Zhiguang. A survey on the visual analytics of multivariate networks[J]. Journal of Computer-Aided Design \& Computer Graphics, 2020, 32(10): 1594-1605(in Chinese)

(刘玉华, 倪璐珊, 周志光. 多元网络可视分析综述 [J]. 计算 机辅助设计与图形学学报, 2020, 32(10): 1594-1605)

[46] Cao N, Lin Y R, Li L Y, et al. g-Miner: interactive visual group mining on multivariate graphs[C] //Proceedings of the 33rd Annual ACM Conference on Human Factors in Computing Systems. New York: ACM Press, 2015: 279-288

[47] Wu Y H, Naveen P, Zhao J, et al. egoSlider: visual analysis of egocentric network evolution[J]. IEEE Transactions on Visualization and Computer Graphics, 2016, 22(1): 260-269

[48] Shen Z Q, Ma K L. Mobivis: a visualization system for exploring mobile data[C] //Proceedings of the IEEE Pacific Visualization Symposium. Los Alamitos: IEEE Computer Society Press, 2008: 175-182

[49] Wang Y, Shi C L, Li L Y, et al. Visualizing research impact through citation data[J]. ACM Transactions on Interactive Intelligent Systems, 2018, 8(1): Article No.5

[50] Yong W, Hammad H, Shi C L, et al. Towards easy comparison of local businesses using online reviews[J]. Computer Graphics Forum, 2018, 37(3): 63-74

[51] Maheswari M U, Sathiaseelan J G R. RankTagViz: a semantic ranking and tags visualization of user travelogues[J]. International Journal of Engineering \& Technology, 2018, 7(2.22): 53-56

[52] Miranda F, Lins L, Klosowski J T, et al. TopKube: a rank-aware data cube for real-time exploration of spatiotemporal data[J]. IEEE Transactions on Visualization and Computer Graphics, 2018, 24(3): 1394-1407 